\title{
Hepatic Endosome Protein Profiling in Apolipoprotein E Deficient Mice Expressing Apolipoprotein B48 but not B100
}

\author{
AnShu Chen, ZhongMao Guo, LiChun Zhou and Hong Yang*
}

Department of Physiology, Meharry Medical College, Nashville, TN

\begin{abstract}
Liver cells absorb apolipoprotein (Apo) B48-carrying lipoproteins in ApoE's absence, albeit not as efficiently as the ApoE-mediated process. Our objective was to identify differentially expressed hepatic endosome proteins in mice expressing ApoB48 but lacking ApoE and ApoB100 expression (ApoE-//B48/48). We purified early and late endosomes from ApoE-/-/B48/48 and wild-type mouse's livers. In ApoE-/-/B48/48 mouse's hepatic endosomes, proteomic analysis revealed elevated protein levels of major urinary protein 6 (MUP), calreticulin, protein disulfide isomerases (PDI) A1, and A3. These proteins are capable of interacting with lipids/lipoproteins and triggering receptormediated endocytosis. In addition, hepatic endosomes from ApoE-/-/B48/48 mice exhibited significantly reduced protein levels of haptoglobin, hemopexin, late endosome/lysosome interacting protein, cell division control protein 2 homolog, Y-soluble Nethylmaleimide- sensitive factor attachment protein, vacuolar ATP synthase catalytic subunit A1, dipeptidyl peptidases II, cathepsin B, D, H, and Z. These proteins participate in plasma heme clearance, receptor-mediated signaling, membrane fusion, endosomal/lysosomal acidification, and protein degradation. The significance of increasing endosomal MUP, calreticulin and PDIs in ApoE-/-/B48/48 mouse liver cells is not clear; however, reducing endosomal/ lysosomal membrane proteins and hydrolases might be, at least partially, responsible for the retarded clearance of plasma ApoB-carrying lipoproteins in ApoE-/-/B48/48 mice.
\end{abstract}

Keywords: Endosomes; Apolipoprotein E; Apolipoprotein B48; Lipoproteins; Proteomics

\section{Introduction}

Apolipoprotein (Apo) E and ApoB100 are two important protein components of the ApoBcarrying lipoproteins. These proteins interact with the low-density lipoprotein receptors (LDLRs) and the LDLR-related proteins (LRP) (Havel and Hamilton, 2004). After binding ApoBcarrying lipoproteins, LDLRs migrate to the plasma membrane's coated pits, where they undergo endocytosis to form endocytic vesicles. The vesicle fuses into the early endosomes, where the low$\mathrm{pH}$ environment triggers bound lipoprotein particle release. LDLRs subsequently return to the cell surface via receptor recycling. The lipoproteins are then transported, within endosomes, to lysosomes, where lipoproteins are degraded (Havel and Hamilton, 2004).

In an ApoE deficiency, the remnants of ApoB100-carrying lipoproteins (e.g. LDL) can still be absorbed by cells through the ApoB100: LDLR interaction. However, the lipoprotein remnants carrying ApoB48 (e.g. chylomicron remnants) do not have a ligand for LDLRs and therefore cannot be internalized via the endocytic pathway mediated by these receptors. These events lead to an increased plasma level of ApoB-carrying lipoproteins. For example, homozygous mutations in human ApoE gene (homozygosity for ApoE2) causes familial type III hyperlipoproteinlemia (Mahley et al., 1999). Similarly, mice deficient in ApoE gene (ApoE-/-) or mice expressing ApoB48 but lacking ApoE and ApoB100 (ApoE-//B48/48) develop hypercholesterolemia due to increased ApoB48-carrying lipoproteins (Piedrahita et al., 1992; Guo et al., 2002; Farese et al., 1996). Interestingly, hypercholesterolemia observed among ApoE-/(Piedrahita et al., 1992; Guo et al., 2002) and ApoE-//B48/48 mice (Farese et al., 1996), fed a normal chow diet, is maintained within narrow limits (between 400 and $600 \mathrm{mg} / \mathrm{dl}$ ), despite the continuous generation of ApoB48-carrying lipoproteins from the intestine and the liver. Apparently, during ApoE's absence there are other pathway(s) meditating the removal of ApoB48-carrying remnants from circulation, albeit not as efficiently as the ApoE-mediated process. There have been studies that demonstrate ApoE-independent pathway(s) mediating the uptake of ApoB48-carrying lipoproteins. For example,
Magoori et al. (2003) reported mice with double deficiencies in ApoE and LRP-5 displayed severe hypercholesterolemia because of increased ApoB48-carrying remnants, as compared to mice lacking ApoE alone (Magoori et al.,2003). However, a LRP-5 deficiency alone had no significant effect on the plasma cholesterol level. These results suggest LRP-5 mediates an ApoE-independent catabolism of plasma lipoprotein remnants. In previous work from our laboratory, we observed that superimposing the heterozygous ataxia-telangiectasiamutated gene (ATM) mutation onto an ApoE deficiency background $(A T M+/ / A p o E-/-)$ increased plasma cholesterol and ApoB48 levels (Wu et al., 2005). In addition, $A T M+/-/ A p o E-/-$ mice showed slower clearance for plasma apoB48-carrying lipoproteins, as compared to the ApoE-/- control mice. However, mice with a heterozygous ATM mutation alone did not significantly increase their plasma cholesterol levels (Wu et al., 2005). These observations suggest ATM plays a role in the ApoE-independent pathway(s) resulting in ApoB48-carrying remnant catabolism.

Previous studies show the liver is the primary organ to remove the ApoE-deficient ApoB48- carrying lipoproteins from circulation (Wu et al., 2005; Yu et al., 2000). To identify proteins specifically involved in ApoE independent mechanisms for ApoB48-carrying lipoprotein endocytosis, we applied a proteomic technique comparing the protein profile of the hepatic endosomes obtained from wild-type mice and ApoE-//B48/48 mice, which excluded ApoE/ApoB100dependent endocytosis. Our results identify differentially expressed

*Corresponding author: Dr. Hong Yang, Department of Physiology, Meharry Medical College, Nashville, TN, Tel: (615) 327-5772; Fax: (615) 3212949; E-mail: hyang@mmc.edu

Received September 10, 2010; Accepted September 23, 2010; Published September 23, 2010

Citation: Chen A, Guo Z, Zhou L, Yang H (2010) Hepatic Endosome Protein Profiling in Apolipoprotein E Deficient Mice Expressing Apolipoprotein B48 but not B100. J Bioanal Biomed 2: 100-106. doi:10.4172/1948-593X.1000031

Copyright: ( $) 2010$ Chen A, et al. This is an open-access article distributed unde the terms of the Creative Commons Attribution License, which permits unrestricted use, distribution, and reproduction in any medium, provided the original author and source are credited. 
proteins from endosomes obtained from $A p o E-/-/ B 48 / 48$ mice and wild-type controls. We also discuss the possible involvement of these proteins in endocytosis.

\section{Materials and Methods \\ Animals}

ApoE-/-/B48/48 and $129 \mathrm{vEv}$ wild-type mice were obtained from Jackson laboratory (Bar Harbor, ME). The ApoE-/-/B48/48 mice were generated by crossbreeding $A p o E-/$ mice with $A p o B 48 / 48$ mice. $A p o B 48 / 48$ mice were generated by Farese, et al. (1996). ApoE-/- mice were generated by Piedrahita et al. (1992). ApoB48/48 and ApoE/- mice were generated using embryonic stem cells from 129-strain mice. ApoE-/-/B48/48 and wild-type mice were fed a chow diet containing approximately $5 \%$ fat and $19 \%$ protein by weight (Harlan Teklad, Madison, WI). At 3-4 months of age, mice were anesthetized as described previously (Guo et al., 2002). After being perfused with ice cold phosphate buffered saline $(25 \mathrm{ml})$, the mouse liver was removed for endosome isolation.

\section{Endosomal fractions isolation}

Mouse liver endosomal fractions were isolated as described by Casciola-Rosen et al. (1992), with modifications. Mouse livers were homogenized in $20 \%(\mathrm{w} / \mathrm{v})$ homogenization buffer containing $0.25 \mathrm{M}$ sucrose, $3 \mathrm{mM}$ imidazole $(\mathrm{pH} 7.4), 1.7 \mathrm{nM}$ antipain, $2 \mathrm{nM}$ leupeptin, and $1 \mathrm{mM}$ phenymethylsulfonyl fluoride. The homogenate was centrifuged sequentially at $460 \mathrm{xg}$ for $10 \mathrm{~min}, 24,000 \mathrm{xg}$ for 8 $\mathrm{min}$, and $100,000 \mathrm{xg}$ for $90 \mathrm{~min}$. The resulting microsomal pellet was resuspended in homogenization buffer $(1 \mathrm{~g}$ of starting liver/ 2 $\mathrm{ml}$ homogenization buffer). Suspension was diluted with an equal volume of $2 \mathrm{M}$ sucrose and successively overlaid with $1 \mathrm{M}, 0.86 \mathrm{M}$, $0.6 \mathrm{M}$, and $0.25 \mathrm{M}$ sucrose solutions. After centrifugation at 100,000 $\mathrm{xg}$ for $3.5 \mathrm{~h}$ (SW 28 rotor, Beckman L7-55), three distinct fractions with an average density of $1.06,1.09$, and $1.12 \mathrm{~g} / \mathrm{ml}$ were obtained. The 1.06 and 1.09 fractions were pooled to yield late endosomes and the $1.12 \mathrm{~g} / \mathrm{ml}$ fraction contained early endosomes. Early endosomes contain the following contaminations (expressed as a percentage of the total homogenate value): $3 \%$ plasma membranes and $1.8 \%$ lysosomes; while the late endosomes were contaminated with $1 \%$ plasma membranes and 0.5\% lysosomes (Casciola-Rosen et al., 1992).

\section{Protein extraction and quantification}

Endosomal proteins were extracted by a dual precipitation procedure. First, endosomal fractions were suspended in $20 \%$ TCA with $20 \mathrm{mM}$ DTT ( $1 \mathrm{~g}$ of starting liver/ $2 \mathrm{ml} 20 \%$ TCA). The suspension was allowed to precipitate on ice for $2 \mathrm{~h}$ and centrifuged at 1000 $\mathrm{xg}$ for $10 \mathrm{~min}$ (Beckman TJ-6R, Beckman Instruments). The resulting pellet was suspended in acetone with $20 \mathrm{mM}$ DTT $(1 \mathrm{~g}$ of starting liver/ $2 \mathrm{ml}$ acetone). Proteins in the suspension were precipitated at $-20^{\circ} \mathrm{C}$ for $4 \mathrm{~h}$ and pelleted by centrifugation at $1000 \mathrm{xg}$ for $5 \mathrm{~min}$. Residual acetone was removed by lyophilization. The protein pellet was solubilized in lysis buffer ( $30 \mathrm{mM}$ Tris, $8 \mathrm{M}$ Urea. $2 \mathrm{M}$ thiourea, $4 \%$ $(\mathrm{w} / \mathrm{v})$ CHAPS, $\mathrm{pH} 8.5)$, sonicated at $100 \mathrm{~W}$ for $30 \mathrm{~s}$, and centrifuged at $25,000 \mathrm{xg}$ for $1 \mathrm{~h}$. Protein supernatant concentrations were measured in triplicate by a 2-D Quant Kit (Amersham Biosciences, Piscataway, $\mathrm{NJ})$.

\section{Cydye labeling and 2-D gel electrophoresis}

CyDye DIGE fluors (Amersham Biosciences) were used to label protein. Twelve protein samples were examined, i.e. three for each early and late endosome samples obtained from $A p o E-/-/ B 48 / 48$ and wild-type mice. Each endosome sample was pooled from three mice.
$250 \mu \mathrm{g}$ protein aliquots were randomly labeled with $200 \mathrm{pmol}$ of either Cy3 or Cy5. A mixed internal standard methodology described by Alban et al. (2003) was used in this study, i.e. $125 \mu$ g protein aliquots from each of the 12 samples were pooled and labeled with 1200 pmol Cy2. Thereafter, $250 \mu \mathrm{g}$ of Cy3- and Cy5-labeled protein sample was mixed with Cy2-labeled sample $(250 \mu \mathrm{g})$. The tripartitelabeled samples were passively rehydrated into a $24-\mathrm{cm}$ immobilized $\mathrm{pH}$ gradient (IPG) strip (pH 4-7) (Amersham Biosciences), followed by simultaneous isoelectric focusing using an Ettan IPGphore II IEF System (Amersham Biosciences). The IPG strips were then placed on top of a 4-16\% gradient SDSpolyacrylamide gels (PAGE). Samples were simultaneously subjected to 2D-electrophoresis, at $5 \mathrm{~W} /$ gel for 30 min followed by $17 \mathrm{~W} / \mathrm{gel}$ for $5 \mathrm{~h}$. The Cy2-, Cy3-, and Cy5- distributed fluorescence on each gel was individually imaged with mutually exclusive excitation/emission wavelengths of 480/530 nm, 520/590 $\mathrm{nm}$ and 620/680 nm using a Typhoon 9410 laser scanner (Amersham Biosciences). Three protein-spot maps were obtained per gel. To pick protein spots of interest, gels were post-stained with SYPRO Ruby (Molecular Probes, Eugene, OR) according to the manufacture's recommendations. SYPRO Ruby image was visualized by $457 / 610 \mathrm{~nm}$ wavelengths using the Typhoon laser scanner.

\section{2-D gel data analysis}

Sample's protein abundance was determined by cy-dye fluorescence intensity. Computer software (DeCyder biological variation analysis module; Amersham Biosciences) was used to simultaneously match all 18 protein-spot maps from 6 gels. Spot abundances were compared by analyzing the ratios between $A p o E$ /-/B48/48 early endosomes and wild-type early endosomes, $A p o E-/-/$ $B 48 / 48$ late endosomes and wild-type late endosomes, $A p o E-/ / B 48 / 48$ late and early endosomes, as well as, between wild-type late and early endosomes. In addition, an unpaired student's $t$-test $p$-value was calculated for each comparison.

\section{In-gel digestion and mass spectrometry analysis}

Proteins of interest were excised with an Ettan Spot Picker (Amersham Biosciences). The gel spots were digested with $15 \mu \mathrm{l}$ porcine modified trypsin protease (Promega, Madison, WI). The tryptic peptides were extracted from the gel plugs, reconstituted in $10 \mu \mathrm{l} 0.1 \%$ trifluoroacetic acid, desalted, and concentrated into $2 \mu \mathrm{l}$ $50 \%$ acetonitrile, $0.1 \%$ trifluoroacetic acid using C18 ZipTip pipette tips (Millipore, Bedford, MA, USA). Peptide eluate $(1 \mu \mathrm{l})$ was mixed with $1 \mu$ lof $\alpha$-cyano-4-hydroxycinnamic acid matrix (Sigma Chemical Co., St. Louis, MO). The mixture was applied to a MALDI target. An overlaid MALDI-TOF-mass spectrometry was performed on a Voyager DE STR (Applied Biosystems, Foster City, CA). Peptide mass maps were acquired in reflectron mode ( $20 \mathrm{KeV}$ accelerating voltage) with 150 ns delayed extraction, averaging 1000 laser shots per spectrum. Trypsin autolytic peptides $(\mathrm{m} / \mathrm{z}=842.51$ and 2211.10 ) were used to internally calibrate each spectrum to within a mass accuracy of $20 \mathrm{ppm}$. Individual ions from each spectrum were inspected for resolution and isotopic distribution. Ions specific for each sample (discrete from background and trypsin-derived ions) were used to interrogate mouse sequences entered in the SWISS-PROT databases using the MASCOT (www.matrixscience.com) database search algorithms. Protein identifications from MALDI-TOF peptide mass maps are based on the tryptic peptide masses.

\section{Western blotting}

Western blot analyzed protein disulfide isomerases (PDIs) and cathepsin B protein levels in whole liver and liver endosomal fractions. 
Mouse liver endosomes were prepared as described above. For preparation of liver total proteins, mouse liver was homogenized in $20 \%(\mathrm{w} / \mathrm{v})$ homogenization buffer containing $20 \mathrm{mM}$ Tris-Cl, $2 \%$ SDS and protease inhibitor cocktail. Homogenates were centrifuged at 14,000 $\mathrm{rpm}, 10 \mathrm{~min}$ at $4^{\circ} \mathrm{C}$. Supernatants containing 5 or $20 \mu \mathrm{g}$ proteins, with same amount of endosomal proteins, were separated on a $10 \%$ SDSPAGE for detection of PDIs and cathepsin B, respectively. Proteins were transferred to polyvinylidene difluoride (PVDF) membranes using a semidry transfer system (Bio-Rad Laboratories, Hercules, CA). After blocking with 5\% BSA, the membrane was immunoblotted with mouse PDIs and cathepsin B antibodies. Membrane was then incubated sequentially with a horseradish peroxidase-conjugated secondary antibody and ECL Plus Western Blotting Detection System (Amersham Biosciences). Protein fluorescence signal was detected using the Typhoon laser scanner and quantified using an image analysis system (ImageQuant, Amersham Biosciences).

\section{Results}

\section{Mouse liver endosome protein profile}

We used 2-D analysis to identify differentially expressed proteins among 4 endosomal samples sets, i.e. early and late endosomes of $A p o E-/-/ B 48 / 48$ and wild-type mice. We generated three protein-spot maps for each endosome line; representative spot maps are shown in Figure 1. By analyzing about 1,000 protein features, a total of 65 protein spots showed differential abundance either between $A p o E$ /-/B48/4 and wild-type early endosomes, between $A p o E-/ / B 48 / 48$ and wild-type late endosomes, between $A p o E-/-/ B 48 / 48$ late and early endosomes, or between wild-type late and early endosomes. Fluorescence intensity ratios illustrated differences in protein levels between endosomes (Tables 1 and 2). Figure 2's 2-D gel image shows differentially expressed protein spots with numbers and letterscorresponding to the entries in Tables 1 and 2.

\section{Single protein displays multiple spots in 2-D gel}

By mass spectrometry analysis, interpretable peptide mass fingerprints were obtained for 50 proteins spots, accounting for 35 individual proteins (Table 1). The multiple spots corresponding for a single protein in a 2-D gelelectrophoresis might represent different post-translational modifications (Witzmann et al., 1994). For example, protein phosphorylation might cause a slight isoelectric point (pI) change, shifting the protein to the acidic end of the $\mathrm{pH}$ gradient gel. Therefore, a spot at the basic end of the isoform string might represent the unmodified protein (Witzmann et al., 1994). In this report, 9 proteins displayed more than one spot in 2-D gels. In most of the cases, multiple spots for a single protein show a similar molecular weight (MW) but a different pI. For example, ApoAI, ApoE, cathepsins D, and $Z$ were present in two spots; while albumin, PDI-A1, and $\mathrm{A} 3$ were found in three spots (Table 1 and Figure 2). Interestingly, modifications such as phosphorylation are known to occur in the aforementioned proteins (Beg et al., 1989; Raftery et al., 2005; Sakai et al., 2003; Faust et al., 1987). In this report, we identified cathepsin $\mathrm{B}$ at three spots, two with MW of about $37 \mathrm{kDa}$ and one spot with a MW of about $25 \mathrm{kDa}$. Cathepsin B could be processed to various sizes via post-translational modifications (Hanewinkel et al., 1987). In addition, we also identified haptoglobin (Hpt) $\beta$-chain four times, as a string of discrete spots with a MW of about $39 \mathrm{kDa}$ (Figure 2, spots 19-22). Previous reports indicate Hpt $\beta$-chain resolves into a complex pattern on 2-D gels, probably due to its various post-translational modifications, such as sialylation and neutral glycosylation (Skehel et al., 2000; Tubbs et al., 2005).

\section{Differentially expressed endosomal proteins between ApoE-/-/ $B 48 / 48$ and wild-type mice}

Among the identified 50 protein spots, 10 were up-regulated in the early and late endosomes of the $A p o E-/ / B 48 / 48$ mice, as
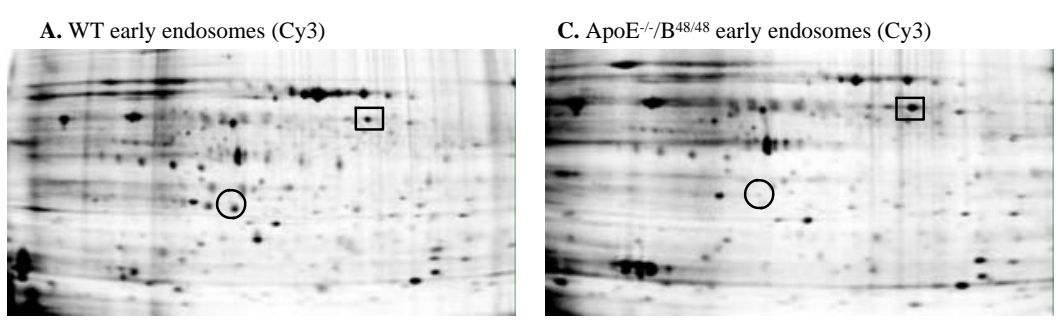

B. WT late endosomes (Cy5)

D. $\mathrm{ApoE}^{-1 / / \mathrm{B}^{48 / 48}}$ late endosomes (Cy5)
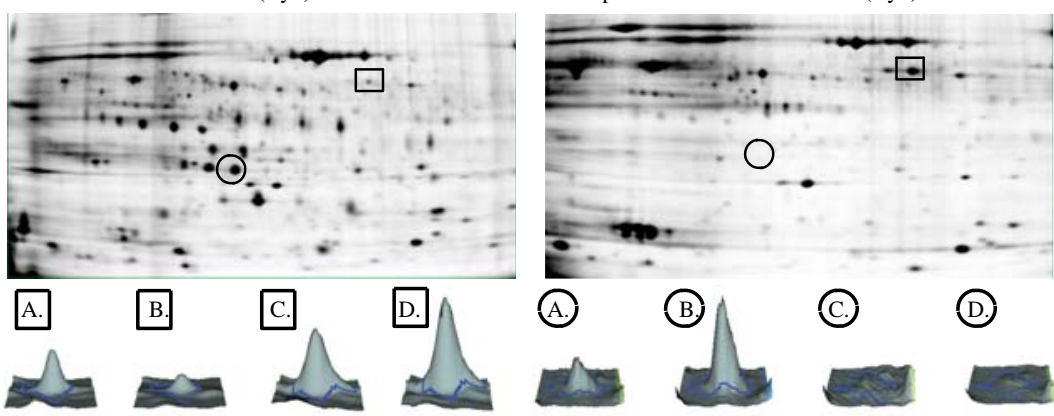

Figure 1: 2-D gel analysis of differentially expressed proteins in hepatic endosomes obtained from ApoE-/-/B48/48 and wild-type mice. Proteins obtained from mouse liver early or late endosomes were labeled with either Cy3 or Cy5 and subjected to 2-D gel electrophoresis with Cy2-labeled pooled standards. Panels A, B, C, and D show representative 2-D gel images of endosomal proteins obtained from ApoE-/-/B48/48 and wild-type (WT) mice. The spot image was mapped at pl ranged from 4 to $7 \mathrm{NL}$ (left to right) and MW ranged from 100 to $10 \mathrm{kDa}$ (top to bottom). Protein-spot maps from all the gel images were matched and the fluorescence spot intensity was compared between endosomes, as detailed in the Materials and Methods. Two representative protein spots, which are differentially expressed among endosomes, are boxed and circled, respectively. The 3-D images show fluorescence distribution of these spots. The 3-D images labeled with boxed or circled letters correspond to the boxed or circled spots in panels A, B, C, and D. Mass spectrometric analysis demonstrates the boxed and the circled spots as PDIA3 and cathepsin B, as shown in Table 1 (spots 11 and 35). 


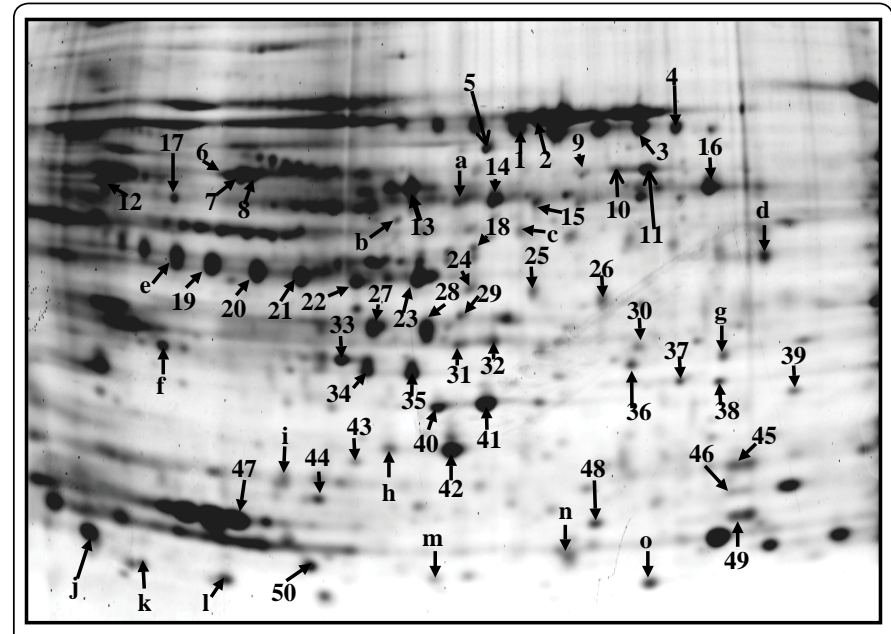

Figure 2: Representative Sypro Ruby-stained gel image shows protein spots of interest. Following DIGE analysis, 2-D gels were post-stained with Sypro Ruby. The protein spots, of which the abundance differed significantly among endosomes, were excised and analyzed with MALDITOF mass spectrometry. Tryptic peptide masses, obtained from the mass spectrometric analysis, were subjected to database searching. Fifty spots were identified and indicated by numbers, which correspond to line entries in Table 1. Fifteen protein spots (indicated by letters) were unidentified due to poor signal in the mass spectrum, low-abundance features, or low molecular weight proteins. Letters correspond to line entries in Table 2.

compared to wild-type controls. Peptide fingerprint analysis indicates these spots correspond to 6 proteins, including ApoAI, peroxiredoxin (prx)-4, major urinary protein 6 (MUP), and three protein chaperons, i.e. calreticulin, PDI-A1, and A3. Interestingly, we observed another protein chaperon, ERp29's abundance decreased in endosomes obtained from ApoE-///B48/48 mice. ERp29 is a endoplasmic reticulum (ER) resident, where its expression profile parallels PDIs (Hubbard et al., 2004). These observations imply increased PDIs in the ApoE/-/B48/48 mouse endosomes are not due to ER contamination. In addition to ERp29, 15 other proteins (corresponding to 22 protein spots) decreased in abundance in ApoE-//B B $8 / 48$ mice's endosomes. These proteins appeared to be more reduced in the late endosomes than in the early endosomes. Some of these proteins are known to be important in the endocytic process. For example, Hpt $\beta$-chain and hemopexin (Hpx) participate in the internalization of plasma free hemoglobin and heme. While late endosome/lysosome interacting protein (p14), cell division control protein 2 homolog (cdc2), and $\gamma$-soluble $\mathrm{N}$-ethylmaleimide-sensitive factor attachment protein $(\gamma$-SNAP) are endocytosis-related signaling proteins. In addition, vacuolar ATP synthase catalytic subunit A1 (V-ATPase A1) is involved in endosomes/lysosomes acidification by transporting protons into these compartments. Down-regulated proteins in $A p o E-/ / B 48 / 48$ mice also include five lysosomal proteases, i.e., dipeptidyl-peptidase II, cathepsins B, D, H, and Z. These enzymes had higher expression levels in the late endosomes than in the early endosomes of wildtype mice. This finding agrees with the general view that lysosomal hydrolase levels are higher in the late endosomes than in the early endosomes. These results also indirectly validate the gradient centrifugation method to separate endosomes. In this report, one spot (Figure 2 and Table 1, spot 40), which corresponded to ApoAI, exhibited opposite regulation in the early and late endosomes of the ApoE-///B48/48 mice, when compared to wild-type controls. Namely, the fluorescence intensity of this spot was greater in the ApoE-/-/ $B 48 / 48$ mice's early endosomes but was lower in the late endosomes of the ApoE-/-/B48/48 mice.

\section{Differentially expressed proteins between early and late endosomes but not between different genotype mice}

As shown in Table 1, 16 spots showed comparable protein abundance in ApoE-/-/B48/48 and wild-type mice, but were significantly different between early and late endosomes of the same genotype. Among these 16 spots, 8 were in higher abundance in the late endosomes than in the early endosomes, while another eight showed a lower abundance in the late endosomes than in the early endosomes. As expected, two spots corresponding to ApoE (spots 31 and 32) disappeared from the $A p o E-/ / B 48 / 48$ endosomal preparation, suggesting the DIGE technique detected ApoE's loss. In wild-type mice, ApoE showed higher levels in the late endosomes than in the early endosomes. This finding is consistent with the notion that late endosomal fraction extracted by gradient centrifugation contains recycling particles, which delivers ApoE and receptors to the cell membrane (Heeren et al., 2001).

\section{Unidentified proteins}

In this report, 15 spots, which differentially expressed among endosomes, were not identified (Table 2) because of poor signal in the mass spectrum above expected background of the trypsinderived ions. Five of the unidentified protein spots were among the lowest-abundant features having Sypro Ruby-image pixel volumes below 1x 108 (Figure 3, spot c, h, i, m, n). However, several spots, such as spots 43 and 46 with similar pixel values,were unambiguous identified (Table 1 and Figure 2) indicating abundance alone does not necessarily correlate with unambiguous identification. Low molecular weight proteins liberate few tryptic peptides simply based on the relative paucity of the cleavage sites. Six of unidentified spots exhibited an apparent MW below $20 \mathrm{kDa}$ (Figure 3; spots j, k, l, m, n, o) and pooling samples from five gels did not provide sufficient material for identification. Limitations of MALDI-derived tryptic mass protein fingerprints likely contribute to ambiguous or no identification of the remaining proteins (Figure 3; spots a, b, d, e, f, g).

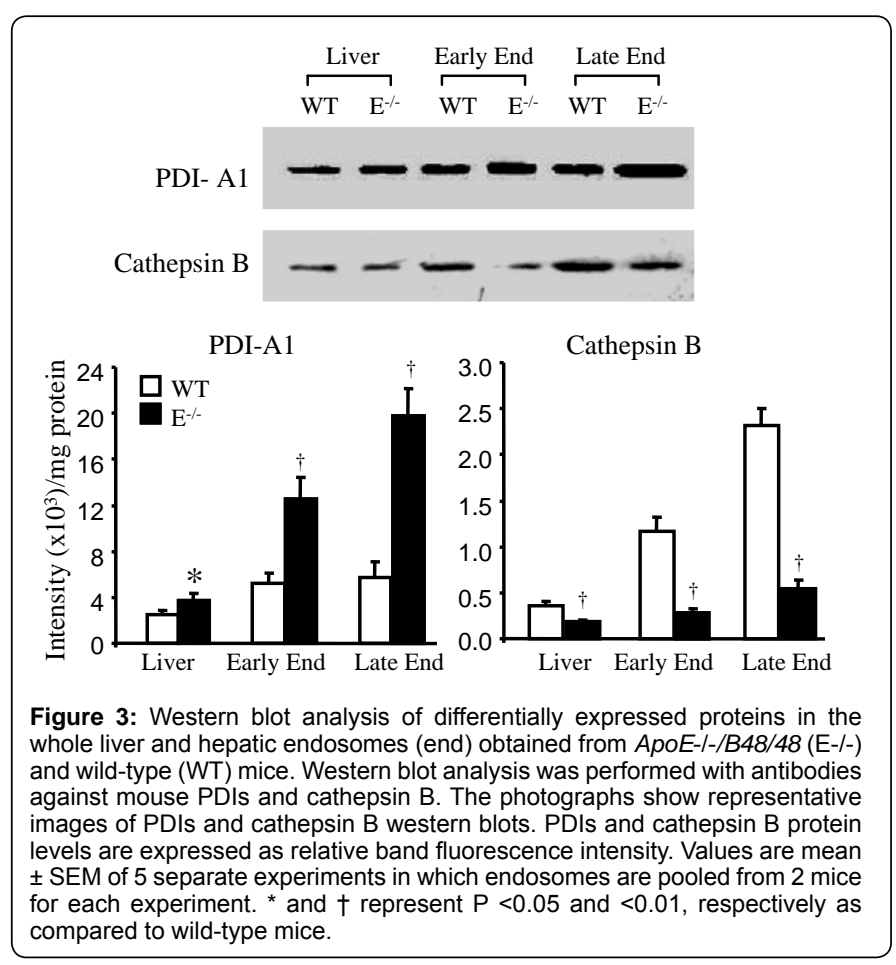




\section{Western blot validation of 2-D DIGE results}

We performed immunoblot experiments to confirm the 2-D gel results, comparing proteins between $A p o E-/ / B 48 / 48$ and wild-type mice. As illustrated in Figure 3, PDI protein levels were significantly increased, while the cathepsin B proteins levels were significantly reduced in endosomes obtained from $A p o E-/ / B 48 / 48$ mice compared to those from wild-type mice. These data are consistent with the 2-D DIGE results (Figure 2 and Table 1). Data in Figure 3 also shows PDI and cathepsin B endosomal protein levels are higher than in the whole liver homogenates, and the whole liver PDI and cathepsin B protein levels mirror endosomal levels, i.e. PDI protein levels are significantly increased, while the cathepsin B protein levels are significantly reduced in the $A p o E-/ / B 48 / 48$ mice.

\section{Discussion}

This study, for the first time, identified a number differentially expressed proteins in the hepatic endosomes obtained from ApoE-/-/ $B 48 / 48$ and wild-type mice. The most important finding is that hepatic endosomes obtained from $A p o E-/ / B 48 / 48$ mice have significantly elevated MUP, calreticulin, PDI-A1, and A3 protein levels. MUP, a protein secreted into bloodstream by the liver, has an ideal structure for binding and transporting hydrophobic molecules. A truncated MUP fraction was recently suggested to be capable of binding long chain fatty acids and is thought to be involved in fatty acid transportation (Kimura et al., 1991). A cell surface receptor for mouse MUP has been identified (Flower, 2000). Calreticulin was first thought to be a $\mathrm{Ca}^{2+}$-binding ER chaperone (Gelebart et al., 2005) but is also found in other membrane-bound organelles, on the cell surface,and in the extracellular environment (Gelebart et al., 2005). Calreticulin can bind to a diverse spectrum of proteinsand can escort associated proteins to the cell surface, eliciting a receptor-mediated endocytosis (Berwin et al., 2004). Receptors that recognize calreticulin include: CD91 (a LDL receptorrelated protein), scavenger receptor class-A (SRA), and SREC-I (scavenger receptor expressed by endothelial cell-I) (Berwin et al., 2004). These receptors also mediate the modified LDL uptake through a variety of cell types (Berwin et al., 2004). In addition, reports suggest calreticulin together with CD91, on the cell surface, provides a binding site for the endocytic processes of virus, bacteria, and apoptotic cells (Ghebrehiwet and Peerschke, 2004). PDIs were also thought to be ER proteins originally, where they catalyzes disulfide bond formation in the newly synthesized proteins (Ellgaard and Ruddock, 2005). In the intestine and liver, PDIs interact with a 97-kd peptide to form a heterodimer microsomal triglyceride transfer protein (MTP), which bind to ApoB100 or ApoB48, in the ApoB-carrying lipoprotein assembly process (Berriot-Varoqueaux et al., 2000). MTP binds to partially degraded remnants of ApoB-containing lipoproteins with a high affinity (Hussain et al., 1997). MTP also is present in the cell membrane and endosomes/lysosomes (Bagshaw et al., 2005). In ApoE-/- mouse's hepatic endosomes, the increased MUP, calreticulin, and PDI protein levels suggests these proteins may participate in hepatic uptake of ApoB48-carrying lipoproteins. It is highly likely that MUP, calreticulin, and PDIs interact with ApoB48-carrying lipoprotein protein or lipid component and then subsequently present these lipoproteins to the cell surface receptors for endocytosis. Definitive experimental data is needed to prove this postulation.

ApoAI is a structural protein of high-density lipoprotein (HDL) particle and exhibits two distinct isoforms with similar molecular weights but different pls, in mouse liver endosomes. We found both isoforms significantly upregulated in early endosomes of $A p o E-/-1$
B48/48 mice, compared to wild-type controls. This data suggests an increased holoparticle endocytosis of HDL in the $A p o E-/ / B 48 / 48$ mouse liver. Previous data indicates HDL holoparticle endocytosis is a pathway for liver cells to remove cholesterol from the circulation (Schwartz et al., 2004). Thus in hypercholesterolemia mouse models increased ApoAI protein levels is not surprising in the early hepatic endosomes. However, two ApoAI isoforms in the late endosomes showed opposite changes in the $A p o E-/ / B 48 / 48$ mouse liver, i.e. the basic isoform increased 1.97-fold, while the acidic isoform decreased 1.8 -fold in the ApoE-//B48/48 mice. Why the basic and acidic ApoAI isoforms are oppositely regulated in the hepatic late endosomes of the ApoE-/-/B48/48 mice remains unknown.

Another important finding is hepatic endosomes obtained from ApoE-/-/B48/48 mice significantly reduce V-ATPase A1 abundance, as well as, five lysosomal proteases and three endocytosis-related signaling proteins, when compared to wild-type controls. The vacuolar ATPases are a family of ATP-driven proton pumps responsible for acidification of intracellular compartments including clathrincoated vesicles, endosomes, lysosomes, Golgi, and secretory vesicles (Forgac, 1998). The acidic endosomal environment is required for internalized ligand-receptor complex dissociation and endosomal carrier vesicles budding, which transfer ligands from early to late endosomes (Forgac, 1998). Vacuolar ATPases are composed of two domains, V0 and V1. The V0 domain is composed of 5 subunits (subunits a-d) and is responsible for proton translocation. The V1 domain is composed of 8 subunits (subunits $\mathrm{A}-\mathrm{H}$ ) and is responsible for ATP hydrolysis. The V-ATPase A1 is a V1 domain subunit (Forgac, 1998). The reduced lysosomal proteases in the ApoE-//B $448 / 48$ mice include dipeptidyl peptidases II, as well as, cathepsin B, D, H, and Z. These lysosomal proteases are synthesized as proenzymes. In mammalian cells, mannose 6-phosphate receptors transport them from the trans-Golgi network to endosomes/lysosomes, where they are converted to mature enzymes (Ishidoh and Kominami, 2002). The reduced signaling proteins in the $A p o E-/ / B 48 / 48$ mice include p14, cdc2, and $\gamma$-SNAP. p14 is associated with the cytoplasmic face of the late endosomes, where it participates in the signaling transduction induced by receptor endocytosis (Qian et al., 2005). $\mathrm{Cdc} 2$ is a protein kinase that phosphorylates proteins involved in cell proliferation. Interestingly, recent studies demonstrate cdc2 also exists in the endocytic compartment of rat livers and is able to phosphorylate proteins that play a fundamental role in membrane traffic regulation and receptor endocytosis (Vergés et al., 1997). $\gamma$ SNAP functions in membrane fusion and is essential not only for the endosome formation, but also for the endosome's interaction with lysosomes and the cell membrane (Stenmark and Zerial, 2001). As the expression of V-ATPase A1, lysosomal proteases, and endocytosisrelated signaling proteins decrease in the endosomes, we suggest this might impair the endocytic process in ApoE-/-/B48/48 mice.

We also report significantly reduced Hpt $\beta$-chain and Hpx protein levels in hepatic endosomes of $A p o E-/ / B 48 / 48$ mice, as compared to wild-type controls. Hpt and Hpx are key plasma hemoproteins that function to remove hemoglobin and heme released from ruptured erythrocytes. In plasma, Hpt-hemoglobin and Hpx-heme complexes initiate receptor-mediated endocytosis in hepatocytes or cells in the hepatic reticuloendothelial system (Anderson and Frazer, 2005). Heme is potentially highly toxic because it can intercalate into lipid membrane/ lipoproteins producing hydroxyl radicals. Hpt- and Hpxdependent heme clearance may be important for atherosclerosis (Skehel et al., 2000). Interestingly, a high-fat diet can elevate plasma Hpt in ApoE3 transgenic mice and in wild-type mice (Skehel et al., 2000). 
Citation: Chen A, Guo Z, Zhou L, Yang H (2010) Hepatic Endosome Protein Profiling in Apolipoprotein E Deficient Mice Expressing Apolipoprotein B48 but not B100. J Bioanal Biomed 2: 100-106. doi:10.4172/1948-593X.1000031

In this report, several proteins, not proven to participate in the plasma transportation and/or hepatic uptake of lipids/lipoproteins, showed significant differences between endosomes obtained from ApoE-//B48/48 and wild-type mice. For example, adenosine kinase (AK), ERp29, indolethylamine N-methyltransferase (INMT), and sulfite oxidase protein levels were significantly reduced in the $A p o E-/ / B 48 / 48$ mice, as compared to the wild-type controls. AK phosphorylates adenosine to adenosine monophosphate. ERp29 is present in folding complexes of several ER secretory proteins, suggesting it may facilitate folding and/or export of secretory proteins in/from the ER (Hubbard et al., 2004). INMT catalyzes tryptamine $\mathrm{N}$-methylation and structurally related compounds. Sulfite oxidase oxidizes sulfites to sulfate. In addition to those down-regulated enzymes, we observed significantly elevated Prx-4 level in the hepatic endosomes of $A p o E-$ /-/B48/48 mice, as compared to wild-type controls. Prxs are novel peroxidases that catalyze the detoxification of various peroxide substrates such as $\mathrm{H} 2 \mathrm{O} 2$, peroxinitrite, and hydroperoxides. Of the six known members of the family, Prx-4 is present as a secretable form in most tissues (Rhee et al., 2005).

In summary, this report applied a proteomic analysis to identify differentially expressed proteins in the hepatic endosomes obtained from $A p o E-/ / B 48 / 48$ and wild-type mice. Our data demonstrates the protein levels of MUP, calreticulin, PDI- A1, and A3 were elevated in ApoE-///B48/48 mice. MUP, calreticulin and PDIs capacity to interact with lipids/lipoproteins and with cell surface receptors suggests these proteins might assist the hepatic uptake of ApoB48-carrying lipoproteins in ApoE's absence. Additionally, ApoE-/-/B48/48 mouse endosomes exhibited a reduction of proteins involved in heme uptake, such as Hpt and Hpx, for endocytosis-related signaling, such as $\mathrm{p} 14$, cdc2 and $\gamma$-SNAP, and endosomal acidification and protein degradation, such as V-ATPase A1, dipeptidyl peptidases II, and cathepsins. Reduction in these proteins might slow the endocytic process, contributing to reduced clearance of ApoB48-carrying lipoproteins in $A p o E-/-/ B 48 / 48$ mice. The 2-D gels analysis used separated proteins with MWs from 10-100 kDa and pls from 4-7 NL. Our data represent only a part of the entire protein spectrum that is differentially expressed in the hepatic endosomes of $A p o E-/-/ B 48 / 48$ and wild-type mice.

\section{Acknowledgements}

The authors thank Dr. David B. Friedman for technical assistance of the proteomics studies. This study is supported by NIH grants: SC1HL101431 (H.Y.), R01HL089382 and R01ES014471 (Z.M.G.).

\section{References}

1. Alban A, David SO, Bjorkesten L, Andersson C, Sloge E, et al (2003) A novel experimental design for comparative two-dimensional gel analysis: twodimensional difference gel electrophoresis incorporating a pooled internal standard. Proteomics 3: 36-44.

2. Anderson GJ, Frazer DM (2005) Hepatic iron metabolism. Semin Liver Dis 25 : 420-432.

3. Bagshaw RD, Mahuran DJ, Callahan JW (2005) A proteomic analysis of lysosomal integral membrane proteins reveals the diverse composition of the organelle. Mol Cell Proteomics 4: 133-143.

4. Beg ZH, Stonik JA, Hoeg JM, Demosky SJ Jr, Fairwell T, et al. (1989) Human apolipoprotein A-I. Post-translational modification by covalent phosphorylation. J Biol Chem 264: 6913-6921.

5. Berriot-Varoqueaux N, Aggerbeck LP, Samson-Bouma M, Wetterau JR (2000) The role of the microsomal triglygeride transfer protein in abetalipoproteinemia. Annu Rev Nutr 20: 663-697.

6. Berwin B, Delneste Y, Lovingood RV, Post SR, Pizzo SV (2004) SREC-I, a type $F$ scavenger receptor, is an endocytic receptor for calreticulin. J Biol Chem 279 : 51250-51257.

7. Casciola-Rosen LA, Renfrew CA, Hubbard AL (1992) Lumenal labeling of rat hepatocyte endocytic compartments. Distribution of several acid hydrolases and membrane receptors. J Biol Chem 267: 11856-11864.

8. Ellgaard L, Ruddock LW (2005) The human protein disulphide isomerase family: substrate interactions and functional properties. EMBO Rep 6: 28-32.

9. Farese RV Jr, Véniant MM, Cham CM, Flynn LM, Pierotti V, et al.(1996) Phenotypic analysis of mice expressing exclusively apolipoprotein B48 or apolipoprotein B100. Proc Natl Acad Sci U S A 93: 6393-6398.

10. Faust PL, Wall DA, Perara E, Lingappa VR, Kornfeld S (1987) Expression of human cathepsin $D$ in Xenopus oocytes: phosphorylation and intracellular targeting. J Cell Biol 105: 1937-1945.

11. Flower DR (2000) Beyond the superfamily: the lipocalin receptors. Biochim Biophys Acta 1482: 327-336.

12. Forgac $M$ (1998) Structure, function and regulation of the vacuolar $(\mathrm{H}+)$ ATPases. FEBS Lett 440: 258-263.

13. Gelebart P, Opas M, Michalak M (2005) Calreticulin, a Ca2+-binding chaperone of the endoplasmic reticulum. Int J Biochem Cell Bio 37: 260-266.

14. Ghebrehiwet B, Peerschke EI (2004) cC1q-R (calreticulin) and gC1q-R/ p33: ubiquitously expressed multi-ligand binding cellular proteins involved in inflammation and infection. Mol Immunol 41: 173-183.

15. Guo Z, Mitchell-Raymundo F, Yang H, Ikeno Y, Nelson J, et al. (2002) Dietary restriction reduces atherosclerosis and oxidative stress in the aorta of apolipoprotein E-deficient mice. Mech Ageing Dev 123: 1121-1131.

16. Hanewinkel H, Glössl J, Kresse $H$ (1987) Biosynthesis of cathepsin B in cultured normal and I-cell fibroblasts. J Biol Chem 262: 12351-12355.

17. Havel RJ, Hamilton RL (2004) Hepatic catabolism of remnant lipoproteins Where the action is. Arterioscler Thromb Vasc Biol 24: 213-215.

18. Heeren J, Grewal T, Jäckle S, Beisiegel U (2001) Recycling of apolipoprotein E and lipoprotein lipase through endosomal compartments in vivo. J Biol Chem. 276: 42333-42338.

19. Hubbard MJ, Mangum JE, McHugh NJ (2004) Purification and biochemical characterization of native ERp29 from rat liver. Biochem J 383: 589-597.

20. Hussain MM, Bakillah A, Jamil H (1997) Apolipoprotein B binding to microsoma triglyceride transfer protein decreases with increases in length and lipidation: implications in lipoprotein biosynthesis. Biochemistry 36: 13060-13067.

21. Ishidoh K, Kominami E (2002) Processing and activation of lysosomal proteinases. Biol Chem 383: 1827-1831.

22. Kimura H, Odani S, Nishi S, Sato H, Arakawa M (1991) Primary structure and cellular distribution of two fatty acid-binding proteins in adult rat kidneys. J Biol Chem 266: 5963-5972.

23. Magoori K, Kang MJ, Ito MR, Kakuuchi H, loka RX, et al. (2003) Severe hypercholesterolemia, impaired fat tolerance, and advanced atherosclerosis in mice lacking both low density lipoprotein receptor-related protein 5 and apolipoprotein E. J Biol Chem 278: 11331-11336.

24. Mahley RW, Huang Y, Rall SC Jr (1999) Pathogenesis of type III hyperlipoproteinemia (dysbetalipoproteinemia). Questions, quandaries, and paradoxes. J Lipid Res 40: 1933-1949.

25. Piedrahita JA, Zhang SH, Hagaman JR, Oliver PM, Maeda N (1992) Generation of mice carrying a mutatnt apolipoprotein $\mathrm{E}$ gene inactivated by gene targeting in embryonic stem cells. Proc Natl Acad Sci U S A 89: 4471-4475.

26. Qian C, Zhang Q, Wang X, Zeng L, Farooq A, et al. (2005) Structure of the adaptor protein $\mathrm{p} 14$ reveals a profilin-like fold with distinct function. $\mathrm{J}$ Mol Biol 347: 309-321.

27. Raftery M, Campbell R, Glaros EN, Rye KA, Halliday GM, et al. (2005) Phosphorylation of apolipoprotein-E at an atypical protein kinase CK2 PSD/E site in vitro. Biochemistry 44: 7346-7353.

28. Rhee SG, Chae HZ, Kim K (2005) Peroxiredoxins: a historical overview and speculative preview of novel mechanisms and emerging concepts in cell signaling. Free Radic Biol Med 38: 1543-1552.

29. Sakai J, Ishikawa H, Kojima S, Satoh H, Yamamoto S, et al. (2003) Proteomic analysis of rat heart in ischemia and ischemia-reperfusion using fluorescence twodimensional difference gel electrophoresis. Proteomics 3: 1318-1324.

30. Schwartz CC, VandenBroek JM, Cooper PS (2004) Lipoprotein cholesteryl ester production, transfer, and output in vivo in humans. J Lipid Res 45: 15941607.

31. Skehel JM, Schneider K, Murphy N, Graham A, Benson GM, et al. (2000) Phenotyping apolipoprotein $\mathrm{E}^{\star} 3$-leiden transgenic mice by two-dimensional 
polyacrylamide gel electrophoresis and mass spectrometric identification. Electrophoresis 21: 2540-2545.

32. Stenmark, H, Zerial M (2001) Molecular mechanisms of membrane fusion in the endocytic pathway. M. Marsh, editor Endocytosis Oxford University Press In., New York 94-110.

33. Tubbs KA, Kiernan UA, Niederkofler EE, Nedelkov D, Bieber AL, et al. (2005) High-throughput MS-based protein phenotyping: application to haptoglobin Proteomics 5: 5002-5007.

34. Vergés M, Castro A, Jaumot M, Bachs O, Enrich C (1997) Cyclin A is present in the endocytic compartment of rat liver cells and increases during liver regeneration. Biochem Biophys Res Commun. 230: 49-53.

35. Witzmann FA, Jarnot BM, Parker DN, Clack JW (1994). Modification of hepatic immunoglobulin heavy chain binding protein (BiP/Grp78) following exposure to structurally diverse peroxisome proliferators. Fundam Appl Toxicol 23: 1-8.

36. Wu D, Yang H, Xiang W, Zhou L, Shi M, et al. (2005) Heterozygous mutation of ataxia-telangiectasia mutated gene aggravates hypercholesterolemia in apoEdeficient mice. J Lipid Res 46: 1380-1387.

37. Yu KC, Jiang Y, Chen W, Cooper AD (2000) Rapid initial removal of chylomicron remnants by the mouse liver does not require hepatically localized apolipoprotein E. J Lipid Res 41: 1715-1727. 